\title{
Estudio de las condiciones de iluminación dentro de las instalaciones de la Facultad de Ingeniería Mecánica y Eléctrica región Poza Rica - Tuxpan
}

\section{Study of lighting conditions within the facilities of the Faculty of Mechanical and Electrical Engineering, Poza Rica - Tuxpan region}

CORTEZ-DOMINGUEZ, Cristóbal†*, RAMOS-GONZALEZ, Luz María, CRUZ-ORDUÑA, María Inés y LAGUNA-CAMACHO, Juan Rodrigo

Universidad Veracruzana, Facultad de Ingeniería Mecánica y Eléctrica, Región Poza Rica - Tuxpan

ID $1^{\mathrm{er}}$ Autor: Cristóbal, Cortez Domínguez / ORC ID: 0000-0002-5720-4405, Researcher ID Thomson: G-2870-2019, CVU CONACYT ID: 972957

ID $1^{\text {er }}$ Coautor: Luz María, Ramos González / ORC ID: 0000-0002-8658-2472, Researcher ID Thomson: G-1385-2019

ID $2^{\text {do }}$ Coautor: María Inés, Cruz Orduña / ORC ID: 0000-0002-8771-0771, CVU CONACYT ID: 347038

ID $3^{\text {er }}$ Coautor: Juan Rodrigo, Laguna Camacho / ORC ID: 0000-0003-0974-5204, CVU CONACYT ID: 100845

DOI: $10.35429 /$ JEE.2019.11.3.7.15

Recibido 20 Septiembre, 2019; Aceptado Diciembre 10, 2019

\begin{abstract}
Resumen
La iluminación es una parte fundamental en el acondicionamiento ergonómico de los puestos de trabajo; si bien, el ser humano tiene la capacidad para adaptarse a las diferentes condiciones lumínicas, la deficiencia en la misma ocasiona fatiga visual, que puede ser una de las causas por la cual los trabajadores cometan errores en sus actividades o incluso hasta provocar accidentes. el presente trabajo se centra en un análisis de las condiciones de iluminación en las aulas, laboratorios y cubículos de la facultad de Ingeniería Mecánica y Eléctrica, e identificar sí, las áreas en donde las tareas visuales asociadas a los puestos de trabajo tienen una iluminación deficiente o excesiva que provoque deslumbramiento, para determinar qué medidas preventivas, o correctivas tomar, con la finalidad de lograr un ambiente seguro y saludable para la comunidad universitaria. Con el apoyo de un luxómetro se realizan las mediciones para calcular la iluminación promedio (EP) y compararlo con la Norma Oficial Mexicana "NOM-025-STPS-2008, Condiciones de iluminación en los centros de trabajo" que establece los límites máximos permisibles (500 luxes). Los resultados obtenidos reflejan que no todas las áreas mencionadas cumplen con la NOM-025-STPS-2008.
\end{abstract}

Iluminación, luxómetro, Seguridad

\begin{abstract}
Lighting is a fundamental part in the ergonomic conditioning of workstations; Although, the human being has the ability to adapt to different light conditions, the deficiency in it causes visual fatigue, which can be one of the causes for which workers make mistakes in their activities or even to cause accidents. The present work focuses on an analysis of the lighting conditions in the classrooms, laboratories and cubicles of the faculty of Mechanical and Electrical Engineering, and identify yes, the areas where the visual tasks associated with the workstations have poor lighting or excessive that causes glare, to determine what preventive or corrective measures to take, in order to achieve a safe and healthy environment for the university community. With the help of a luxometer measurements are made to calculate the average illumination (EP) and compare it with the Official Mexican Standard "NOM-025-STPS-2008, Conditions of lighting in the workplace" that establishes the maximum permissible limits ( 500 luxes). The results obtained reflect that not all the mentioned areas comply with NOM-025STPS-2008.
\end{abstract}

Lighting, Luxmeter, Security

Citación: CORTEZ-DOMINGUEZ, Cristóbal, RAMOS-GONZALEZ, Luz María, CRUZ-ORDUÑA, María Inés y LAGUNA-CAMACHO, Juan Rodrigo. Estudio de las condiciones de iluminación dentro de las instalaciones de la Facultad de Ingeniería Mecánica y Eléctrica región Poza Rica - Tuxpan. Revista de Ingeniería Eléctrica. 2019. 3-11: 7-15

\footnotetext{
$\dagger$ Investigador contribuyendo como primer Autor.

*Correspondencia al Autor correo electrónico: (ccortez@uv.mx)
} 


\section{Introducción}

Cuando se habla de ergonomía, se entiende que se trata de un conjunto de teorías y conocimientos que adapta los productos, tareas, herramientas, espacios y el entorno en general a las capacidades y necesidades de las personas.

Las personas, por tanto, son el objetivo principal de estudio de la ergonomía, por ello, sus análisis son las reacciones, capacidades y habilidades de los trabajadores, de manera que se pueda diseñar su entorno y elementos de trabajo ajustados a estas capacidades y que se consigan unas condiciones óptimas de confort y de eficacia productiva.

La ergonomía trata de ajustar y adaptar el entorno de trabajo a las características y capacidades del trabajador. La ergonomía busca mejorar la eficiencia, seguridad y bienestar de los trabajadores. [Luis Rosel Ajamil, et al (2005)]

De acuerdo con la ergonomía, existen diferentes problemáticas en un espacio laboral, tanto físicos como morales, por ejemplo:

Las condiciones ambientales del puesto de trabajo: iluminación, ruido, temperatura, vibraciones, etc.

El diseño del puesto de trabajo: alturas de trabajo, espacio disponible, herramientas utilizadas, etc.

La carga física realizada: posturas forzadas, movimientos repetitivos, manejo manual de cargas, fuerzas, etc.

Los aspectos mentales o psicosociales del trabajo: descanso, presión de tiempos, participación en las decisiones, relaciones entre compañeros y con los responsables, entre otros. [Luis Rosel Ajamil, et al (2005)]

\section{Historia de la iluminación}

El hombre prehistórico descubre el fuego y con éste muchos beneficios comienzan a llegar como la iluminación, cocción de alimentos, calor, entre otros. En Roma, lo que se utilizaba como iluminación fueron las lámparas de aceite, que se colgaban al techo con una cadena, que a lo largo del tiempo se iban decorando con labrados y ornamentos de metal. [Diego Fraga, 2013]
Más tarde, en la Edad Media, aparecieron otros tipos de iluminación, como las linternas con pabilos internos. A principios de 1860, aparecen en Estados Unidos las lámparas de querosén, derivado del petróleo.

Del otro lado del mundo, el químico inglés Sir Humphry Davy inventó la primera lámpara eléctrica de arco provocando la incandescencia de un hilo fino de platino en el aire al aplicar tensión en sus extremos para que circulase corriente. Basándose en los descubrimientos de Davy, el francés Foucault desarrolló una lámpara de arco, que por descarga eléctrica entre dos electrodos de carbón producía luz. [Diego Fraga, 2013]

En 1901 se inventa la lámpara de vapor de mercurio que es la precursora de la lámpara fluorescente. Just y Haran fabricaron en 1906 una lámpara con filamento de tungsteno que reemplazaba al de carbono. Un año más tarde se sustituyen con filamentos de wolframio a los de tungsteno, y en 1913 se fabricaron las primeras bombillas incandescentes rellenadas con gas.

En 1911 Georges Claude inventó en Francia la lámpara de neón. En 1927 se patentó la lámpara fluorescente. Los bulbos de las lámparas fluorescentes están recubiertos en la parte interior para maximizar la eficiencia. [Diego Fraga, 2013]

\section{Niveles de iluminación para tareas visuales y áreas de trabajo}

Los niveles mínimos de iluminación que deben incidir en el plano de trabajo, para cada tipo de tarea visual o área de trabajo se muestran en la tabla 1, a continuación, donde relaciona la tarea visual del puesto del trabajo, con el área de trabajo y los niveles mínimos de iluminación.

\begin{tabular}{|l|l|l|}
\hline \multicolumn{1}{|c|}{$\begin{array}{c}\text { Tarea Visual del } \\
\text { puesto de Trabajo }\end{array}$} & \multicolumn{1}{|c|}{ Área de Trabajo } & \multicolumn{1}{|c|}{$\begin{array}{c}\text { Niveles } \\
\text { mínimos de } \\
\text { iluminación } \\
\text { (luxes) }\end{array}$} \\
\hline $\begin{array}{l}\text { En exteriores: } \\
\text { distinguir el área de } \\
\text { tránsito, desplazarse } \\
\text { caminando, } \\
\text { vigilancia, } \\
\text { movimiento de } \\
\text { vehículos. }\end{array}$ & $\begin{array}{l}\text { Exteriores generales: } \\
\text { patios y estacionamientos }\end{array}$ & \\
\hline $\begin{array}{l}\text { En exteriores: } \\
\text { distinguir el área de } \\
\text { tránsito, desplazarse } \\
\text { caminando, } \\
\text { vigilancia, } \\
\text { movimiento }\end{array}$ & $\begin{array}{l}\text { Interiores de generales: } \\
\text { almacenes de poco } \\
\text { movimiento, pasillos, } \\
\text { escaleras, } \\
\text { estacionamientos } \\
\text { cubiertos, labores en minas } \\
\text { subterráneas, iluminación } \\
\text { de emergencia }\end{array}$ & \\
\hline
\end{tabular}

CORTEZ-DOMINGUEZ, Cristóbal, RAMOS-GONZALEZ, Luz María, CRUZ-ORDUÑA, María Inés y LAGUNA-CAMACHO, Juan Rodrigo. Estudio de las condiciones de iluminación dentro de las instalaciones de la Facultad de Ingeniería Mecánica y Eléctrica región Poza Rica Tuxpan. Revista de Ingeniería Eléctrica. 2019 


\begin{tabular}{|c|c|c|}
\hline En interiores & $\begin{array}{l}\text { Áreas de circulación y } \\
\text { pasillos; salas de espera, } \\
\text { salas de descanso, cuartos } \\
\text { de almacén, plataformas, } \\
\text { cuartos de calderas }\end{array}$ & 100 \\
\hline $\begin{array}{l}\text { Recubrimiento visual } \\
\text { simple: inspección } \\
\text { visual, recuento de } \\
\text { piezas, trabajo en } \\
\text { banco y máquina }\end{array}$ & $\begin{array}{lr}\begin{array}{l}\text { Servicios al } \\
\text { almacenaje }\end{array} & \begin{array}{r}\text { personal: } \\
\text { rudo, }\end{array} \\
\text { recepción y } & \text { despacho, } \\
\text { casetas de vigilancia, } \\
\text { cuartos de compresores y } \\
\text { pailería }\end{array}$ & 200 \\
\hline $\begin{array}{l}\text { Distinción moderada } \\
\text { de detalles: ensamble } \\
\text { simple, trabajo medio } \\
\text { en banco y máquina, } \\
\text { inspección simple, } \\
\text { empaque y trabajos } \\
\text { de oficina. }\end{array}$ & $\begin{array}{l}\text { Talleres: áreas de empaque } \\
\text { y ensamble, aulas y } \\
\text { oficinas }\end{array}$ & 300 \\
\hline $\begin{array}{lr}\text { Distinción clara de } \\
\text { detalles: maquinado y } \\
\text { acabado delicados, } \\
\text { ensamble } \\
\text { inspección } \\
\text { moderadamente } \\
\text { difícil, captura y } \\
\text { procedimiento de } \\
\text { información, manejo } \\
\text { de instrumentos y } \\
\text { equipo de laboratorio. }\end{array}$ & $\begin{array}{l}\text { Talleres de precisión: salas } \\
\text { de cómputo, áreas de } \\
\text { dibujo, laboratorios. }\end{array}$ & 500 \\
\hline 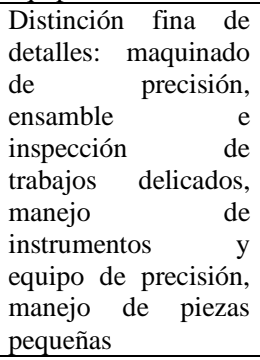 & $\begin{array}{l}\text { Talleres de alta precisión: } \\
\text { de pintura y acabado de } \\
\text { superficies y laboratorios } \\
\text { de control de calidad. }\end{array}$ & 750 \\
\hline $\begin{array}{l}\text { Alta exactitud en la } \\
\text { distinción de detalles: } \\
\text { ensamble, proceso e } \\
\text { inspección de piezas } \\
\text { pequeñas } \\
\text { complejas, acabado } \\
\text { con pulidos finos }\end{array}$ & $\begin{array}{l}\text { Proceso: ensamble e } \\
\text { inspección de piezas } \\
\text { complejas y acabados con } \\
\text { pulidos finos }\end{array}$ & 1,000 \\
\hline $\begin{array}{l}\text { Alto grado de } \\
\text { especialización en la } \\
\text { distinción de detalles }\end{array}$ & \begin{tabular}{ll}
\multicolumn{3}{l}{ Proceso de gran exactitud. } \\
Ejecución de tareas \\
visuales: & \\
- & \multicolumn{2}{l}{ De bajo contraste y } \\
tamaño & muy \\
pequeño & por \\
periodos & \\
prolongados & \\
- & $\begin{array}{l}\text { Exactas y muy } \\
\text { prolongadas, y }\end{array}$ \\
- & $\begin{array}{l}\text { Muy especiales de } \\
\text { extremadamente } \\
\text { bajo contraste y } \\
\text { pequeño tamaño }\end{array}$
\end{tabular} & 2,000 \\
\hline
\end{tabular}

Tabla 1 Niveles permisibles de iluminación Fuente: NORMA Oficial Mexicana NOM-025-STPS-2008, Condiciones de iluminación en los centros de trabajo.

Se debe realizar un recorrido por todas las áreas del centro de trabajo donde los trabajadores realizan sus tareas visuales, y considerar, en su caso, los reportes de los trabajadores, así como recabar la información técnica. [NORMA Oficial Mexicana NOM-025STPS-2008]
La norma también indica que para determinar las áreas y tareas visuales de los puestos de trabajo debe recabarse y registrarse la información del reconocimiento de las condiciones de iluminación de las áreas de trabajo, así como de las áreas donde exista una iluminación deficiente $\mathrm{o}$ se presente deslumbramiento $\mathrm{y}$, posteriormente, conforme se modifiquen las características de las luminarias o las condiciones de iluminación del área de trabajo, con los datos siguientes:

a. Distribución de las áreas de trabajo, del sistema de iluminación (número y distribución de luminarias), de la maquinaria y del equipo de trabajo;

b. Potencia de las lámparas;

c. Descripción del área iluminada: colores y tipo de superficies del local o edificio;

d. Descripción de las tareas visuales y de las áreas de trabajo, de acuerdo con la Tabla Niveles permisibles de iluminación;

e. Descripción de los puestos de trabajo que requieren iluminación localizada.

De acuerdo con la información obtenida durante el reconocimiento, se establecerá la ubicación De los puntos de medición de las áreas de trabajo seleccionadas, donde se evaluarán los niveles de iluminación. [NORMA Oficial Mexicana NOM-025-STPS-2008]

Así mismo, cuando se utilice iluminación artificial, antes de realizar las mediciones, se debe de cumplir con lo siguiente:

a. Encender las lámparas con antelación, permitiendo que el flujo de luz se estabilice; si se utilizan lámparas de descarga, incluyendo lámparas fluorescentes, se debe esperar un periodo de 20 minutos antes de iniciar las lecturas. Cuando las lámparas fluorescentes se encuentren montadas en luminarias cerradas, el periodo de estabilización puede ser mayor;

b. En instalaciones nuevas con lámparas de descarga o fluorescentes, se debe esperar un periodo de 100 horas de operación antes de realizar la medición, y 
c. Los sistemas de ventilación deben operar normalmente, debido a que la iluminación de las lámparas de descarga y fluorescentes presentan fluctuaciones por los cambios de temperatura.

De acuerdo con la NORMA Oficial Mexicana NOM-025-STPS-2008, las áreas de trabajo se deben dividir en zonas del mismo tamaño, de acuerdo a lo establecido con el índice de área (IC) mostrado en la columna A (número mínimo de zonas a evaluar) de la Tabla 2, y realizar la medición en el lugar donde haya mayor concentración de trabajadores o en el centro geométrico de cada una de estas zonas; en caso de que los puntos de medición coincidan con los puntos focales de las luminarias, se debe considerar el número de zonas de evaluación de acuerdo a lo establecido en la columna B (número mínimo de zonas a considerar por la limitación) de la Tabla Número de Zonas de Medición en relación al índice de área.

En caso de coincidir nuevamente el centro geométrico de cada zona de evaluación con la ubicación del punto focal de la luminaria, se debe mantener el número de zonas previamente definido.

\begin{tabular}{|c|c|c|}
\hline $\begin{array}{l}\text { Índice de } \\
\text { área (IC) }\end{array}$ & $\begin{array}{c}\text { A) } \\
\text { Número } \\
\text { mínimo de } \\
\text { zonas a evaluar }\end{array}$ & $\begin{array}{c}\text { B) } \\
\text { Número de } \\
\text { zonas a } \\
\text { considerar por } \\
\text { la limitación }\end{array}$ \\
\hline $\mathrm{IC}<1$ & 4 & 6 \\
\hline $1 \leq \mathrm{IC} \leq 2$ & 9 & 12 \\
\hline $2 \leq \mathrm{IC} \leq 3$ & 16 & 20 \\
\hline $3 \leq \mathrm{IC}$ & 25 & 30 \\
\hline
\end{tabular}

Tabla 2 Número mínimo de zonas a evaluar Fuente: NORMA Oficial Mexicana NOM-025-STPS-2008, Condiciones de iluminación en los centros de trabajo

El valor del índice de área, para establecer el número de zonas a evaluar, está dado por la ecuación 1.

$I C=\frac{(x)(y)}{h(x+y)}$

Dónde:

$I C=$ Índice del área.

$x, y=$ dimensiones del área (largo y ancho), en metros.

$h=$ altura de la luminaria respecto al plano de trabajo, en metros.

\section{Espacio 1: Aula de clases}

La iluminación es de suma importancia para el desempeño tanto de los alumnos como del personal al realizar sus labores si tenemos una iluminación inadecuada puede tener impacto directo en la calidad del trabajador o estudiante tanto como en su salud, esta práctica tiene como objetivo que el alumno comprenda y pueda hacer un análisis en aulas como en Laboratorios. A continuación se muestra el desarrollo del análisis de iluminación en el aula de clases C2M, cabe mencionar que éste es uno de los salones con capacidad para 30 estudiantes.

\section{Pasos:}

Paso 1: Medición del largo, ancho y alto del aula C2M. Ésta se realiza de forma física y con ayuda de un flexómetro intentando tomar la medición más exacta posible, esto se muestra en la figura 1 , donde se indica la forma correcta de iniciar la medición del aula.

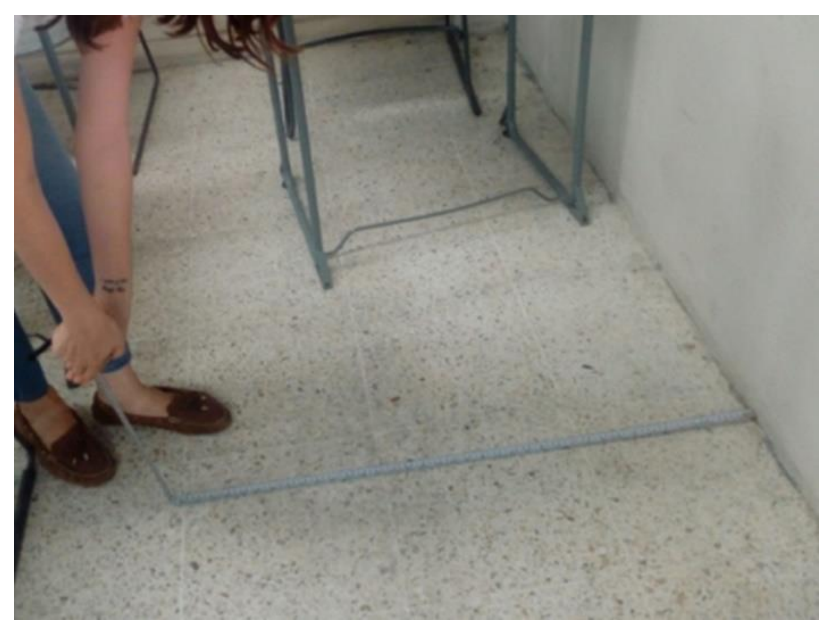

Figura 1 Medición en el aula de clases C2M de la Facultad de Ingeniería Mecánica y Eléctrica

Fuente: Elaboración Propia

De este ejercicio, se obtuvieron las siguientes mediciones:

$$
\begin{aligned}
& \mathrm{x}=6.35 \mathrm{~m} \text { (largo) } \\
& \mathrm{y}=7.71 \mathrm{~m} \text { (ancho) } \\
& \mathrm{h}=2.15 \mathrm{~m} \text { (altura) }
\end{aligned}
$$

Paso 2. Cálculo del índice de área (IC): para realizar el cálculo del índice de área (IC) se recurre a la ecuación 1 y se sustituyen los valores, esto se desglosa en el siguiente apartado. 


$$
\begin{aligned}
& I C=\frac{(x)(y)}{h(x+y)} \\
& I C=\frac{(6.35 \mathrm{~m})(7.71 \mathrm{~m})}{2.15 \mathrm{~m}(6.35 \mathrm{~m}+7.71 \mathrm{~m})}=\frac{(48.95) \mathrm{m}^{2}}{(30.22) \mathrm{m}^{2}} \\
& \therefore \mathrm{IC}=1.619
\end{aligned}
$$

Paso 3: Se ubica en la tabla 2 el número de zonas a considerar, la cual se hace referencia en la figura 2, con la que se puede observar que como el índice de área está entre 1 y 2 el número mínimo de zonas a evaluar es 9 y el número de zonas a considerar por la limitación es 12 .

\begin{tabular}{|c|c|c|}
\hline $\begin{array}{c}\text { Índice de } \\
\text { área (IC) }\end{array}$ & $\begin{array}{c}\text { A) } \\
\text { Número } \\
\text { mínimo de } \\
\text { zonas a evaluar }\end{array}$ & $\begin{array}{c}\text { B) } \\
\text { Número de } \\
\text { zonas a } \\
\text { considerar por } \\
\text { la limitación }\end{array}$ \\
\hline $\mathrm{IC}<1$ & 4 & 6 \\
\hline $1 \leq \mathrm{IC} \leq 2$ & 9 & 12 \\
\hline $2 \leq \mathrm{IC} \leq 3$ & 16 & 20 \\
\hline $3 \leq \mathrm{IC}$ & 25 & 30 \\
\hline
\end{tabular}

Figura 2 Identificación del número de zonas para un índice de área de 1.6

Fuente: NORMA Oficial Mexicana NOM-025-STPS-2008, Condiciones de iluminación en los centros de trabajo

Paso 4: Se realiza la medición con un luxómetro en la posición E1 ésta indica que la fotocelda del luxómetro debe estar colocada de cara a la superficie, esto se muestra en la figura 3 , donde se puede observar claramente la posición del luxómetro (instrumento de color rojo) y el sensor de luz (instrumento color negro) éste último es el que se encarga de tomar el valor de luminosidad mientras que el luxómetro lo transforma en una escala visible en el display que tiene incluido.

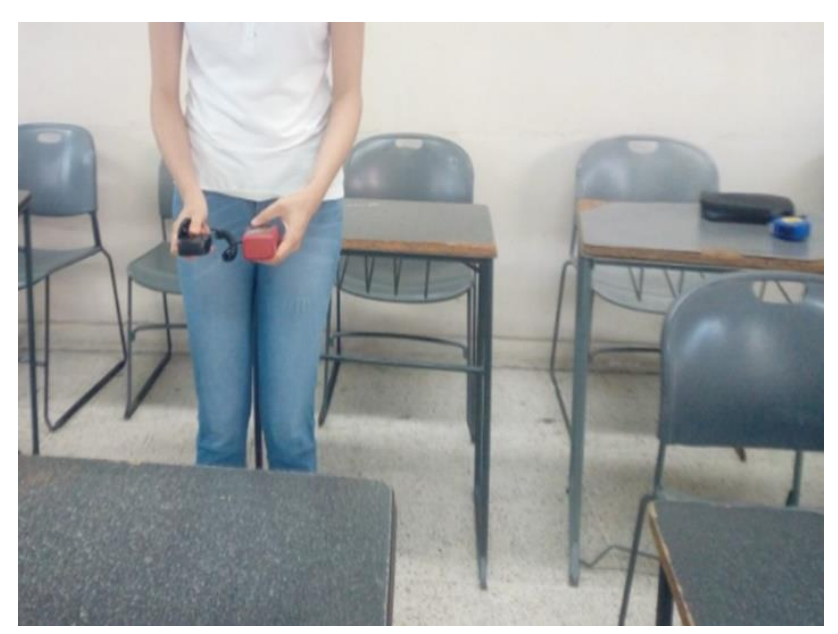

Figura 3 Luxómetro en posición E1

Fuente: Elaboración Propia
Por otro lado, en la imagen 4 se muestra la posición E2 del luxómetro, esto con el objetivo de tomar en cuenta la reflexión y refracción de la luz aproximadamente en el mismo punto de análisis.

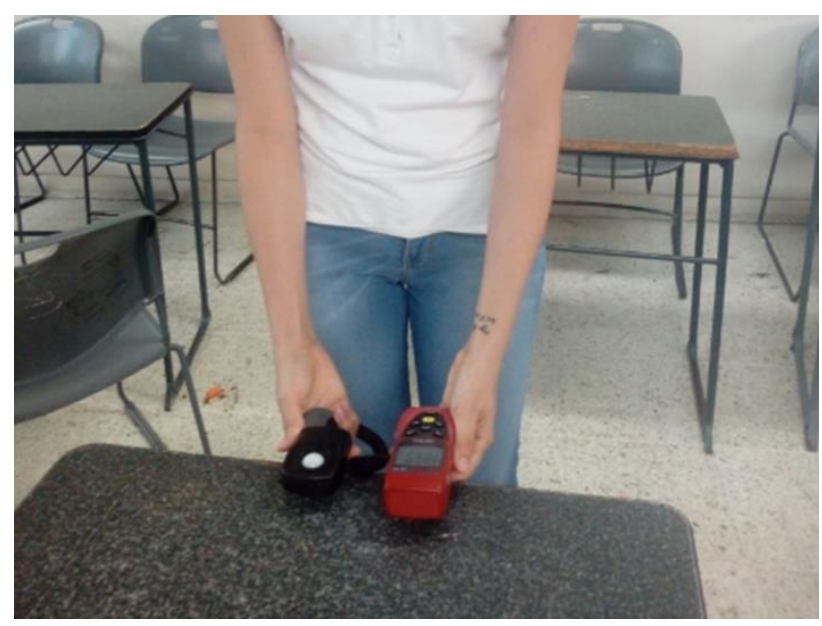

Figura 4 Luxómetro en posición E2 Fuente: Elaboración Propia

Paso 5: Determinar el Factor de Reflexión de Superficie Kf. Para determinar este factor, se recurre a la ecuación 2, donde relaciona los valores obtenidos de las posiciones E1 y E2 vistas anteriormente.

$\mathrm{Kf}=\frac{E 1}{E 2}(100)$

A continuación, se desarrolla la ecuación con los valores que se tienen de E1 y E2 para cada una de las nueve áreas a analizar indicada por los subíndices consecutivos en cada ecuación.

$$
\begin{aligned}
\mathrm{Kf}_{1} & =\frac{162}{386}(100)=0.4196(100)=41.96 \\
\mathrm{Kf}_{2} & =\frac{142}{360}(100)=.3944(100)=39.44 \\
\mathrm{Kf}_{3} & =\frac{150}{409}(100)=0.3667(100)=36.67 \\
\mathrm{Kf}_{4} & =\frac{112}{248}(100)=0.4516(100)=45.16 \\
\mathrm{Kf}_{5} & =\frac{115}{352}(100)=0.3267(100)=32.67 \\
\mathrm{Kf}_{6} & =\frac{128}{338}(100)=0.3786(100)=37.86 \\
\mathrm{Kf}_{7} & =\frac{105}{300}(100)=0.35(100)=35 \\
\mathrm{Kf}_{8} & =\frac{97}{342}(100)=0.2836(100)=28.36 \\
\mathrm{Kf}_{9} & =\frac{133}{324}(100)=0.4104(100)=41.04
\end{aligned}
$$

CORTEZ-DOMINGUEZ, Cristóbal, RAMOS-GONZALEZ, Luz María, CRUZ-ORDUÑA, María Inés y LAGUNA-CAMACHO, Juan Rodrigo. Estudio de las condiciones de iluminación dentro de las instalaciones de la Facultad de Ingeniería Mecánica y Eléctrica región Poza Rica Tuxpan. Revista de Ingeniería Eléctrica. 2019 
Paso 6: Obtención del nivel promedio. La iluminación promedio se obtiene mediante la ecuación 3, la cual referencia todos los valores tomados de E en las mediciones anteriores (Ei) y el número total de mediciones.

$$
\begin{aligned}
& E p=\frac{1}{18}(\Sigma \mathrm{Ei}) \\
& E p=\frac{1}{18}(162+386+142+360+\cdots) \\
& E p=0.0555(4203) \\
& \therefore E p=233.26 \text { Luxes }
\end{aligned}
$$

Este valor se compara con la tabla de referencia en la tabla 1, la cual se menciona en las conclusiones.

\section{Espacio 2: Laboratorio}

La iluminación en lo que respecta al área industrial debe tener presente un gran número de luminarias ya que deben abarcar espacios muy grandes y extensos, también deben poseer características distintas a luminarias convencionales o residenciales como poseer mayor potencia, brillo, incandescencia y aceptar los cambios bruscos de voltaje.

Para esto es necesario analizar la tarea visual a desarrollar y determinar la cantidad y tipo de iluminación que proporcione el máximo rendimiento visual y cumpla con las exigencias de seguridad y comodidad como también seleccionar el equipo de alumbrado que proporcione la luz requerida de la manera satisfactoria. Al igual que en el ejercicio anterior, se realizan paso a paso el análisis de la iluminación ahora en el laboratorio de cómputo.

Paso 1: Medición del largo, ancho y alto del laboratorio. Ésta se realiza de forma física y con ayuda de un flexómetro intentando tomar la medición más exacta posible, esto se muestra en la figura 5.

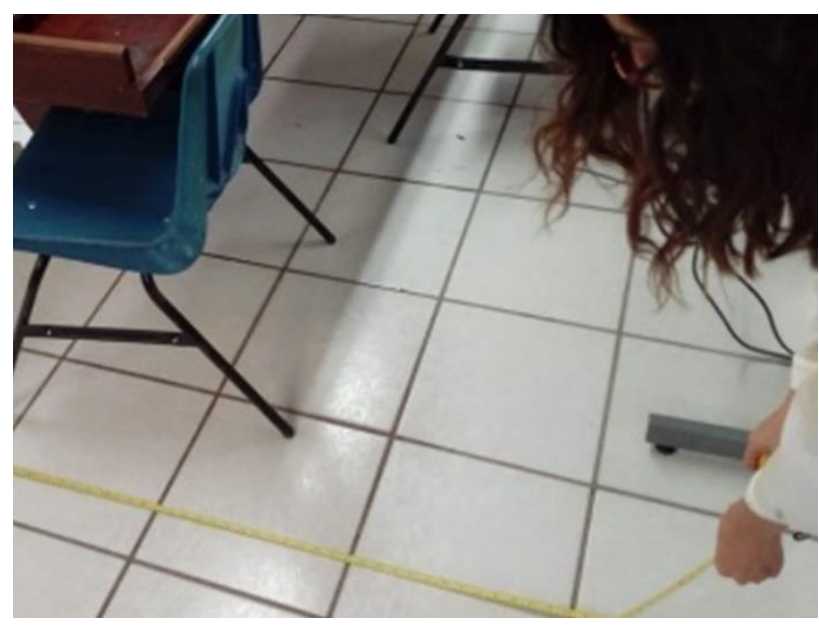

Figura 5 Medición de las dimensiones físicas del laboratorio de cómputo de la FIME

Fuente: Elaboración Propia

De este ejercicio se obtienen los siguientes valores:

$\mathrm{x}=9.5 \mathrm{~m}$

$\mathrm{y}=7.90 \mathrm{~m}$

$\mathrm{h}=2.15 \mathrm{~m}$

Paso 2. Tal como se mostró en el ejercicio anterior, el paso 2 se refiere al cálculo del Índice de área para poder determinar cuántas mediciones se van a realizar.

$$
\begin{aligned}
& I C=\frac{(x)(y)}{h(x+y)} \\
& I C=\frac{(9.5 \mathrm{~m})(7.90 \mathrm{~m})}{2.15 \mathrm{~m}(9.5 \mathrm{~m}+7.90 \mathrm{~m})}=\frac{(75.05)}{(37.41)} \\
& \therefore I C=2.006
\end{aligned}
$$

Paso 3. De acuerdo con la tabla 2, se identifica el número zonas a analizar dentro del espacio de análisis, en este caso, el laboratorio, esto se muestra en la figura 6.

\begin{tabular}{|c|c|c|}
\hline $\begin{array}{c}\text { Índice de } \\
\text { área (IC) }\end{array}$ & $\begin{array}{c}\text { A) } \\
\text { Número } \\
\text { mínimo de } \\
\text { zonas a evaluar }\end{array}$ & $\begin{array}{c}\text { B) } \\
\text { Número de } \\
\text { zonas a } \\
\text { considerar por } \\
\text { la limitación }\end{array}$ \\
\hline $\mathrm{IC}<1$ & 4 & 6 \\
\hline $1 \leq \mathrm{IC} \leq 2$ & 9 & 12 \\
\hline $2 \leq \mathrm{IC} \leq 3$ & 16 & 20 \\
\hline \hline $3 \leq \mathrm{IC}$ & 25 & 30 \\
\hline
\end{tabular}

Figura 6 Identificación del número de zonas para un índice de área de 2.006

Fuente: NORMA Oficial Mexicana NOM-025-STPS-2008, Condiciones de iluminación en los centros de trabajo 
Paso 4: Se realiza la medición con luxómetro en la posición E1 y E2 tal como se describieron en el primer ejercicio, esto lo muestran las figuras 7 y 8 .

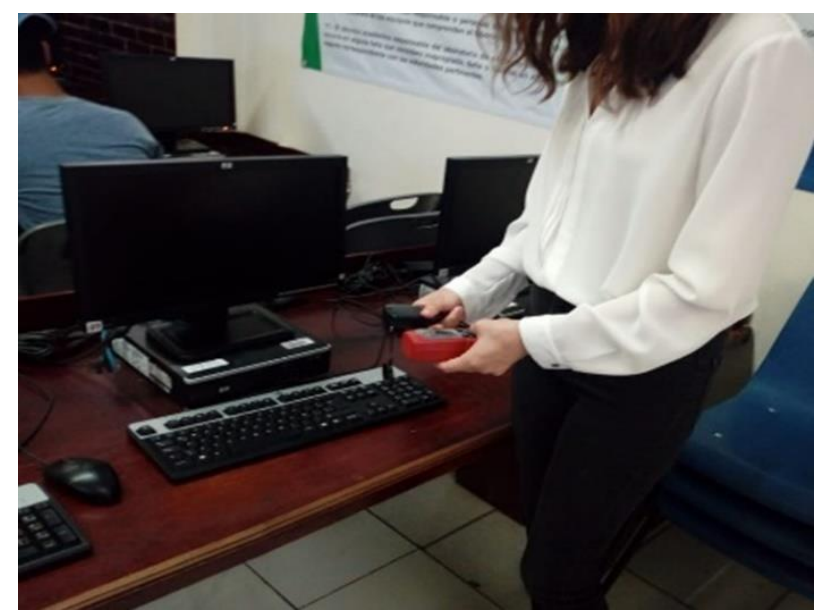

Figura 7. Medición obtenida en posición E1 Fuente: Elaboración Propia

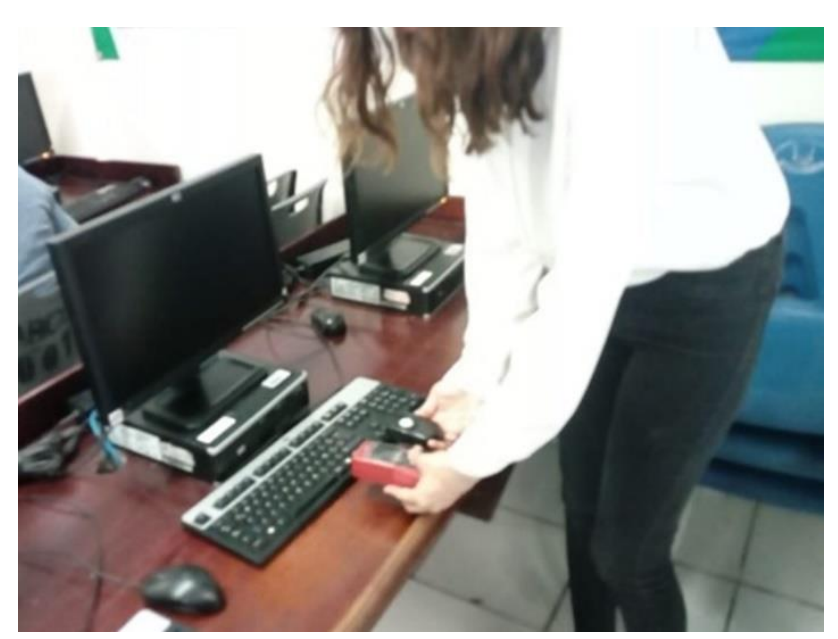

Figura 8 Medición en posición E2

Fuente: Elaboración Propia

Paso 5: Determinar el Factor de Reflexión de Superficie Kf, como en esta caso, la comparación con la tabla 2 mostró que deben ser 16 zonas las que se evaluarían, por tanto, serían 16 valores de Kf que se obtendrían.

$$
\begin{aligned}
\mathrm{Kf}_{1} & =\frac{50}{175}(100)=0.2857(100)=28.57 \\
\mathrm{Kf}_{2} & =\frac{65}{288}(100)=0.2256(100)=22.56 \\
\mathrm{Kf}_{3} & =\frac{30}{170}(100)=0.1764(100)=17.64 \\
\mathrm{Kf}_{4} & =\frac{35}{240}(100)=0.1458(100)=14.58 \\
\mathrm{Kf}_{5} & =\frac{33}{173}(100)=0.1907(100)=19.07 \\
\mathrm{Kf}_{6} & =\frac{44}{220}(100)=0.2(100)=20
\end{aligned}
$$

$$
\begin{aligned}
& \mathrm{Kf}_{7}=\frac{45}{170}(100)=0.2647(100)=26.47 \\
& \mathrm{Kf}_{8}=\frac{46}{226}(100)=0.2035(100)=20.35 \\
& \mathrm{Kf}_{9}=\frac{29}{187}(100)=0.1550(100)=15.50 \\
& \mathrm{Kf}_{10}=\frac{35}{265}(100)=0.1320(100)=13.20 \\
& \mathrm{Kf}_{11}=\frac{32}{170}(100)=0.1882(100)=18.82 \\
& \mathrm{Kf}_{12}=\frac{24}{192}(100)=0.125(100)=12.5 \\
& \mathrm{Kf}_{13}=\frac{52}{192}(100)=0.2708(100)=27.08 \\
& \mathrm{Kf}_{14}=\frac{41}{255}(100)=0.1607(100)=16.07 \\
& \mathrm{Kf}_{15}=\frac{23}{183}(100)=0.1256(100)=12.56 \\
& \mathrm{Kf}_{16}=\frac{32}{240}(100)=0.1333(100)=13.33
\end{aligned}
$$

Paso 6: Obtención del nivel promedio. La iluminación promedio se obtiene mediante la ecuación 3 y cuyo resultado se desarrolla a continuación.

$$
\begin{aligned}
& E p=\frac{1}{32}(50+175+65+288+\cdots) \\
& E p=0.03125(3756) \\
& \therefore E p=117.375 \text { Luxes }
\end{aligned}
$$

Este valor se compara con la tabla de referencia en la tabla 1, la cual se menciona en las conclusiones.

\section{Espacio 3. Cubículo}

Cualquier actividad que se realice requiere que la iluminación sea la correcta. En la iluminación es importante conocer el aspecto cuantitativo (la cantidad de luz que se necesita para realizar el trabajo) y el cualitativo (el impacto que tiene en el usuario de estos espacios). En estos aspectos la luz debe tener componentes de radiación difusa y directa cuya resultante produzcan sombras suaves que nos permitan percibir la forma y posición de los objetos. Al igual que en los dos ejercicios anteriores, se siguen los pasos para el análisis de iluminación en el cubículo de Profesor de Tiempo Completo. 
Paso 1: Medición del largo, ancho y alto del laboratorio. Ésta se realiza de forma física y con ayuda de un flexómetro intentando tomar la medición más exacta posible, esto se muestra en la figura 9 .

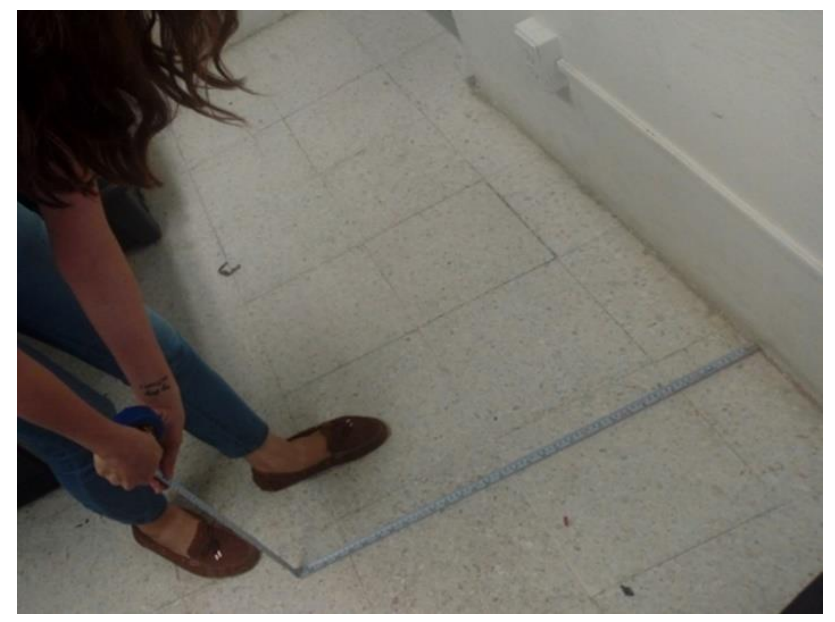

Figura 9 Medición de las dimensiones físicas del cubículo Fuente: Elaboración Propia

Del ejercicio anterior, se obtienen los siguientes valores:

$$
\begin{aligned}
& \mathrm{x}=2.7 \mathrm{~m} \\
& \mathrm{y}=3.82 \mathrm{~m} \\
& \mathrm{~h}=2.10 \mathrm{~m}
\end{aligned}
$$

Paso 2. Tal como se mostró en los ejercicios anteriores, el paso 2 se refiere al cálculo del Índice de área para poder determinar cuántas mediciones se van a realizar.

$I C=\frac{(x)(y)}{h(x+y)}$

$\mathrm{IC}=\frac{(2.7 \mathrm{~m})(3.82 \mathrm{~m})}{2.10 \mathrm{~m}(2.7 \mathrm{~m}+3.82 \mathrm{~m})}=\frac{(10.54)}{(13.81)}$

$I C=0.76$

Paso 3. De acuerdo con la tabla 2, se identifica el número zonas a analizar dentro del espacio de análisis, en este caso, el laboratorio, esto se muestra en la figura 10.

\begin{tabular}{|c|c|c|}
\hline $\begin{array}{c}\text { Índice de } \\
\text { área (IC) }\end{array}$ & $\begin{array}{c}\text { A) } \\
\text { Número } \\
\text { mínimo de } \\
\text { zonas a evaluar }\end{array}$ & $\begin{array}{c}\text { B) } \\
\text { Número de } \\
\text { zonas a } \\
\text { considerar por } \\
\text { la limitación }\end{array}$ \\
\hline $\mathrm{IC}<1$ & 4 & 6 \\
\hline $1 \leq \mathrm{IC} \leq 2$ & 9 & 12 \\
\hline $2 \leq \mathrm{IC} \leq 3$ & 16 & 20 \\
\hline $3 \leq \mathrm{IC}$ & 25 & 30 \\
\hline
\end{tabular}

Figura 10 Identificación del número de zonas para un índice de área de 0.76

Fuente: NORMA Oficial Mexicana NOM-025-STPS-2008, Condiciones de iluminación en los centros de trabajo

Paso 4: Se realiza la medición con luxómetro en la posición E1 y E2 tal como se describieron en el primer ejercicio, esto lo muestran las figuras 11 y 12 .

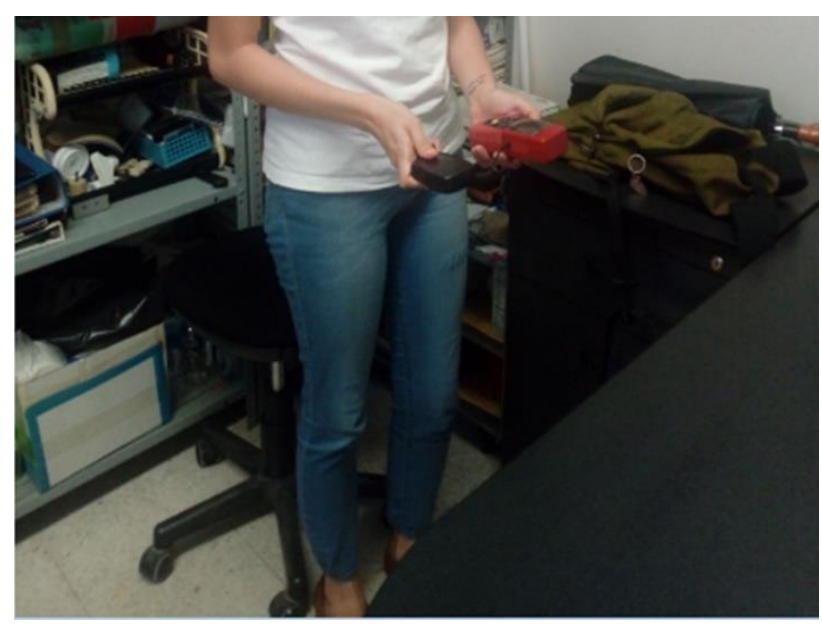

Figura 11 Medición obtenida en posición E1 Fuente: Elaboración Propia

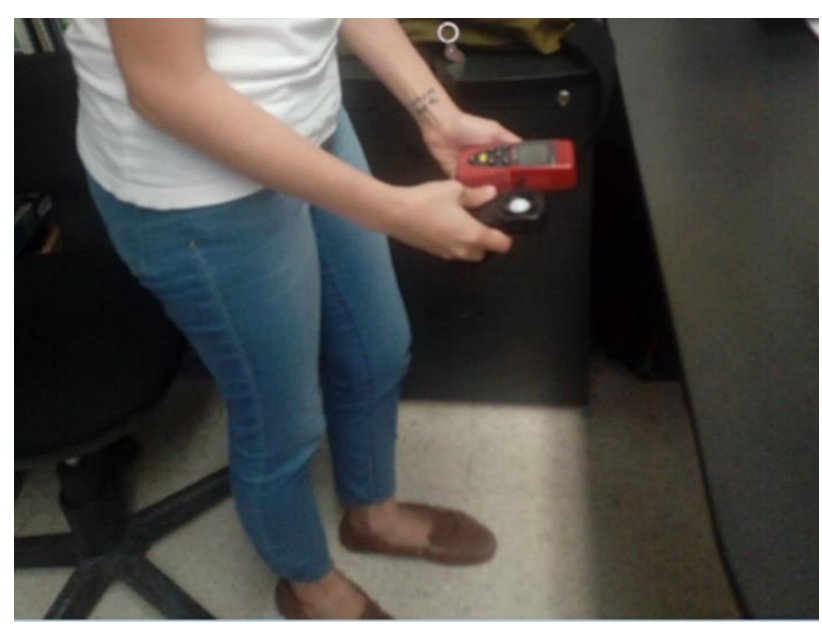

Figura 12. Medición obtenida en posición E2 Fuente: Elaboración Propia 
Paso 5: Determinar el Factor de Reflexión de Superficie Kf, como en esta caso, la comparación con la tabla 2 mostró que deben ser 4 zonas las que se evaluarían, por tanto, serían 4 valores de Kf que se obtendrían

$$
\begin{aligned}
\mathrm{Kf}_{1} & =\frac{31.8}{403}(100)=0.0789(100)=7.89 \\
\mathrm{Kf}_{2} & =\frac{24.4}{276}(100)=0.0884(100)=8.84 \\
\mathrm{Kf}_{3} & =\frac{29.4}{436}(100)=0.0674(100)=6.74 \\
\mathrm{Kf}_{4} & =\frac{34.3}{328}(100)=0.1045(100)=10.45
\end{aligned}
$$

Paso 6: Obtención del nivel promedio. La iluminación promedio se obtiene mediante la ecuación 3 y cuyo resultado se desarrolla a continuación.

$$
\begin{aligned}
& E p=\frac{1}{8}(31.8+408+24.4+276+\cdots) \\
& E p=0.125(1562.9) \\
& \therefore E p=195.36 \text { Luxes }
\end{aligned}
$$

\section{Conclusiones}

Del espacio 1. Dada la condición de luz Natural en los horarios de entre 17:00 y 19:00 horas de exposición a las que fue sometido el análisis, se percató que los lúmenes emitidos por las lámparas en el aula $\mathrm{C} 2 \mathrm{M}$ son deficientes ya que los límites máximos permitidos por la norma son de 300 lux, y los datos obtenidos fueron de 233.26 lux, esto debido a la deficiencia en su potencia lumínica de las lámparas y a la carencia de luz natural.

Del espacio 2. Dada la condición de luz Natural en los horarios de entre 17:00 y 19:00 horas de exposición a las que fue sometido el análisis, se percató que los lúmenes emitidos por las lámparas en el cubículo son deficientes ya que los límites máximos permitidos por la norma son de 500 lux ,y los datos obtenidos fueron de 117.375 lux, esto debido a la deficiencia en su potencia lumínica de las lámparas y a la carencia de luz natural.
Del espacio 3. Dada la condición de luz Natural en los horarios de entre 15:00 y 17:00 horas de exposición a las que fue sometido el análisis , se percató que los lúmenes emitidos por las lámparas en el cubículo son deficientes ya que los límites máximos permitidos por la norma son de 300 lux ,y los datos obtenidos fueron de 195.3625 lux, dando como resultado un error de 104.6375 lux, o en un 34.87 valor porcentual, esto debido a la deficiencia en su potencia lumínica de las lámparas y a la carencia de luz natural.

\section{Referencias}

Fraga, D. (2013). Fraga Iluminación. Obtenido de https://www.fragailuminacion.com.ar/publicaci ones/historia-de-la-iluminacion/

Rodríguez Gómez, A. L. (2018). Manual de prácticas de apoyo a la experiencia educativa de ergonomía. Poza Rica, Veracruz: Tesis, Universidad Veracruzana.

Rosel Ajamil, L. (2005). Manual de ergonomía en la construcción, Fundación laboral en la construcción. Obtenido de http://www.lineaprevencion.com/ProjectMiniSi tes/IS33/cap1.htm 